\title{
Luz Sarmiento Bendezú
}

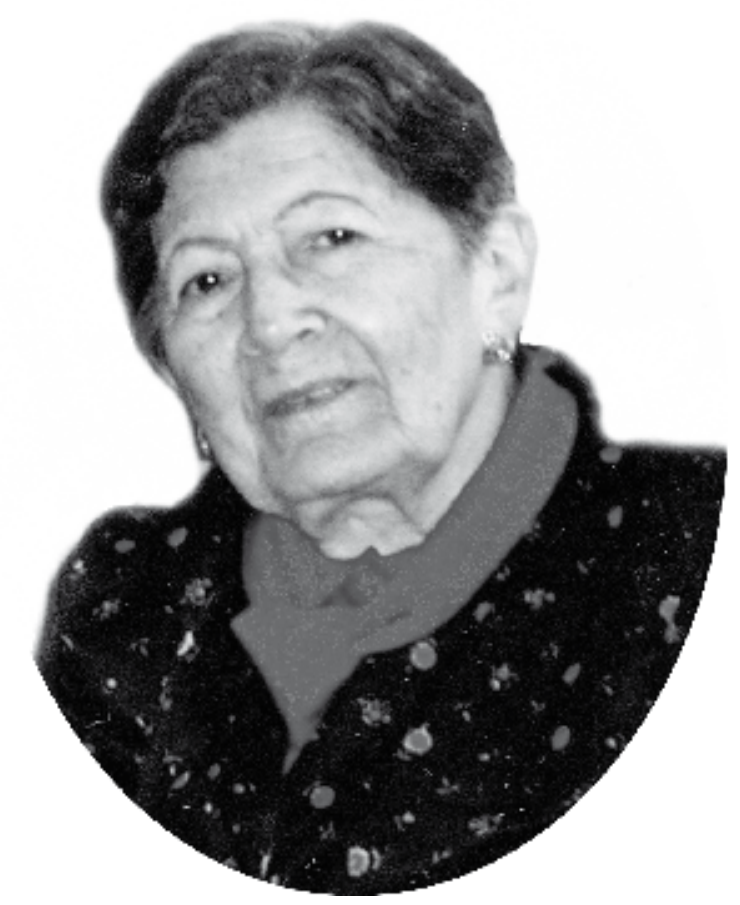

Luz Sarmiento Bendezú nació en Nazca, Ica, el 10 de enero de 1924. El camino que describieron sus actos refleja el decidido espíritu académico que la ha alentado siempre y aún proyecta a sus colegas y alumnos.

La doctora Luz ingreso a la Facultad de Ciencias de Universidad Nacional Mayor de San Marcos en 1942. Su interés e iniciación en el estudio de los parásitos los reafirmó con la tesis para Bachiller en Ciencias Biológicas "Identificación de Haemonchus contortus en carneros nacidos en la sierra del Perú" sustentada en 1946. Ese mismo año empezó su carrera docente como Jefe de Prácticas del curso Zoología Sistemática I en la Facultad de Ciencias de la UNMSM. En 1952 inició sus estudios de Maestría y participó como Asistente del curso de Parasitología del Dpto. del Biología de la Virginia Polytechnick Institute and State University, USA. En 1953 obtuvo el grado de Master of Science con la tesis "Gigantorhinchus ortizi n. sp. of Acanthocephalian from Metachyrus nudicaudatus". En 1954 obtuvo el grado de Doctora en Ciencias Biológicas, en la UNMSM, con la tesis: "Descripción de Paratractis hystrix n. gen., n. sp. de Podocnemis dumeriliana". Continúo desde 1954 hasta 1958 como profesora de Biología, Anatomía y Zoología en el Brenau College en Gainesville, Georgia, USA. En 1959 de regreso al Perú continuó su carrera docente como Catedrática Asociada y asumió el curso de Zoología Sistemática I y en 1970 fue nombrada Jefa de la Sección Helmintología del Museo de Historia Natural de la UNMSM.

Su dedicación y calidad académica le proporcionaron la confianza de sus colegas y autoridades, como prueba de esto fue elegida Jefa del Departamento de Ciencias Biológicas desde 1977 a 1980, periodo durante el cual la universidad peruana se organizaba en el sistema departamentalista, y en 1980 fue elegida Directora del Instituto de Investigación de Ciencias Biológicas Antonio Raimondi (ICBAR).
La doctora Luz fue discípula del Dr. Luis Gonzales-Mugaburu con quién realizó diversas investigaciones en fauna doméstica. Organizó el laboratorio de Helmintología del MHN y en él inició la Colección Helmintológica, poco a poco fue adentrándose en investigaciones parasitológicas de la fauna silvestre, durante su trayectoria profesional participó en la elaboración de catálogos de helmintos en colaboración con investigadores nacionales. Su especialidad fueron los nematodos aunque estudió otros grupos de parasitos llegando a describir 6 nuevas especies tambien realizo estudios de los protozoarios de vida libre en los Pantanos de Villa, donde describió tres nuevas especies.

Su labor se proyectó sobre la comunidad científica peruana. Fue miembro fundador de la Sociedad Peruana de Parasitología (SOPEPA) y entre 1964 y 1966 y 1987 y 1990 fue Vicepresidente de la misma. También fue miembro de la Federación Latinoamericana de Parasitología y de la American Society of Parasitologists.

La importancia de esta labor y su dedicación fue reconocida por la comunidad científica y académica. En el año 1983 el Ministerio de Salud y el Colegio de Biólogos del Perú le concedieron el Diploma de Honor en Reconocimiento al Aporte a la Investigación Científica y Pionera de las Ciencias Biológicas.

En año 1996 la Sociedad Peruana de Parasitología le otorgó el Diploma de Honor por sus valiosos aportes en el campo de la Parasitología y en virtud a su destacada labor científica y académica en la Universidad Nacional Mayor de San Marcos.

Por último nuestra Universidad en el año 2000 la nombra Profesora Emérita en reconocimiento a los méritos y logros del decidido espíritu académico de la doctora Luz Sarmiento. 


\section{Especies descritas por la doctora Luz Sarmiento (se informa el hospedero y la publicación) ${ }^{1}$}

Teleurysorchis gumercindae Gonzales \& Sarmiento, 1990. Hospedero: Schedophilus haedrichi Chirichigno, 1973 «cojinova del norte» o «mocosa». Revista de Ciencias (Lima) 75(1): $39-46$.

Hedruris juninensis Sarmiento, 1977. Hospedero: Batrachophrynus brachydactylus Peters, 1873 «rana». Revista de Ciencias (Lima) 70(1): 45-51.

Stenurus australis Sarmiento \& Tantaleán, 1991. Hospedero: Phocoena spinipinnis Burmeister, 1865 «marsopa espinosa». Publicaciones del Museo de Historia natural UNMSM (A) 36: 1-4.24.

Acanthobothrium gonzalesmugaburoi [sic!] Severino \& Sarmiento, 1979. Hospedero: Myliobatis peruvianus Garman, 1913 «raya águila». Revista de Ciencias (Lima) 71(1): 38-43.

Troglostrongylus wilsoni Sarmiento \& Stough, 1956. Hospedero: Lynx rufus rufus (Schreber, 1777) «lince rojo» o "gato montés». Journal of Parasitology 42(1): 45-48.

Gigantorhynchus ortizi Sarmiento, 1954. Hospedero: Metachirus nudicaudatus (Desmarest, 1817) «rata marsupial de cuatro ojos». Journal of Parasitology 40(4): 448-452.

\section{Especies dedicadas a la doctora Luz Sarmiento ${ }^{1}$}

Pseudoeurysorchis sarmientoi [sic!] Tantaleán, 1974. Hospedero: Seriolella violacea Guichenot, 1848 «cojinova». Revista brasileira de Biologia 34(2): 253-257.

Acanthobothrium lusarmientoi [sic!] Severino \& Verano, 1980. Hospedero: Psammobatis caudispina Hildebrand, 1946 «raya espinosa». Revista de Ciencias (Lima) 72(1): 21-27.

${ }^{1}$ Morales E., L. Sarmiento, L. Sánchez, D. Floríndez y G. Lamas. 2005. Material tipo de helmintos en el Museo de Historia Natural, Universidad Nacional Mayor de San Marcos, (MUSM), Lima, Perú. Rev. peru. biol. 12(3): 463- 472. 